\title{
Propuestas de mejora de las tecnologías de la información y de la comunicación en la formación médica continuada
}

\author{
Luis Rodríguez-Padial, Antonio Medina-Rivilla, María Luz Cacheiro-González
}

Introducción. Tanto la educación como la medicina se han visto transformadas por las tecnologías de la información y de la comunicación (TIC), que deben desempeñar un importante papel en la formación médica continuada (FMC). No obstante, las TIC tienen importantes limitaciones que deben conocerse y corregirse para conseguir una mayor implantación y eficacia docente de éstas. Nuestro objetivo fue conocer la opinión de los usuarios sobre la mejora de las TIC cuando se aplican a la FMC.

Sujetos y métodos. Se utiliza un cuestionario de Internet alojado en la página web de la Fundación Signo con la intención de conocer la opinión de los médicos usuarios y sus propuestas de mejora de las TIC en la FMC.

Resultados. Se ha obtenido información de 661 profesionales sanitarios, 56,7\% de ellos varones. La edad media es de 48,9 años. La falta de interacción entre alumno y profesor (30,3\%), la poca objetividad de la evaluación de los conocimientos adquiridos (18,2\%), la falta de acreditación de los cursos $(17,1 \%)$ y la poca intervención del alumno (11,8\%) son las limitaciones más importantes señaladas. Por tanto, el establecimiento de un buen contacto entre alumno-profesor mejorando la tutoría $(21,8 \%)$ y la promoción de una mayor interacción del alumno durante el proceso de aprendizaje $(20,4 \%)$ son las estrategias de mejora propuestas.

Conclusiones. Este colectivo propone una mejora de la interacción entre profesor-alumno como la principal estrategia para mejorar la eficacia docente de las TIC en la FMC.

Palabras clave. Docencia. Formación médica continuada. Técnicas de información y comunicación.

Improvement proposals of information and communication technology in continuing medical education

Introduction. Both education and medicine have been transformed by information technology (IT), which should play an important role in continuing medical education (CME). However, IT has important limitations that should be known and corrected for greater teacher effectiveness and implementation thereof. Therefore, our aim was to seek feedback from users about IT and its proposals for improving them.

Subjects and methods. A questionnaire on the Internet hosted on the website of the Signo Foundation was used with the intention of getting feedback from users and their suggestions for improvement of IT in CME.

Results. Information from 661 healthcare professionals, 56.7\% of whom were male, was obtained. The average age is 48.9 years. The lack of interaction between student and teacher (30.3\%), lack of objectivity of the evaluation of the knowledge acquired (18.2\%), no accreditation of courses (17.1\%) and low intervention of students (11.8\%) are the most important limitations of IT observed. Therefore, establishing good student-teacher contact $(21.8 \%)$ and promoting greater student interaction during the learning process (20.4\%) are proposed strategies for improvement.

Conclusions. This group has proposed an enhanced teacher-student interaction as the main strategy to improve the teaching effectiveness of IT in CME.

Key words. Continuous medical education. Information technologies. Teaching.

\section{Introducción}

Las tecnologías de la información y la comunicación (TIC) han contribuido al desarrollo de la sociedad de la información y, consecuentemente, al enorme cambio social experimentado con ella. Su impacto ha sido relevante tanto en la educación [1] como en medicina. En esta última, las TIC desempeñan un papel esencial en gestión y seguimiento de pacientes, con lo que contribuyen a mejorar la eficiencia [2,3]; no obstante, su papel en medicina se extiende también a la formación médica $y$, de
Servicio de Cardiología; Complejo Hospitalario de Toledo (L. RodríguezPadial). Departamento de Didáctica, Organización Escolar y Didácticas Especiales; Facultad de Educación; UNED (A. Medina-Rivilla, M.L. CacheiroGonzález); Madrid, España.

Correspondencia:

Dr. Luis Rodríguez Padial. Servicio de Cardiología. Complejo Hospitalario de Toledo. Avda. Barber, 30.

E-45004 Toledo.

E-mail:

Irpadial@gmail.com

Agradecimientos:

Al llustre Colegio Oficial de Médicos de Toledo y a la Fundación Signo, por su colaboración en la realización de la encuesta. Al Dr. Francisco Martín Rodríguez, por su apoyo en el análisis estadístico.

Conflicto de intereses: No declarado.

Competing interests: None declared.

(c) $2015 \mathrm{FEM}$ 
forma notoria, a la formación médica continuada (FMC) [4,5], que según algunos autores ha sido un aspecto relevante y poco tratado hasta la actualidad [6]. En este contexto, el uso de las TIC se ve restringido porque los médicos tienen un escaso conocimiento y realizan un uso limitado de éstas [7-11].

La FMC es al mismo tiempo una obligación ética y un derecho para el médico, especialmente importante en la actualidad, donde los cambios en la medicina son tan rápidos y relevantes. Por ello, es fundamental establecer estrategias que permitan mantener una actualización con la mayor eficiencia y eficacia posibles.

Hemos estudiado previamente el grado de conocimiento y uso de las TIC para el aprendizaje de la electrocardiografía [12] y para su uso en FMC y se ha podido comprobar la actitud de los médicos sobre el uso de estas técnicas y la necesidad de establecer estrategias para su mejora. Parece fundamental que, cuando se diseñen estas estrategias dirigidas a mejorar las TIC en el contexto de la formación médica -y en especial de la FMC-, se considere la opinión que tengan sobre ellas quienes están destinados a utilizarlas, así como las estrategias que propondrían para su mejora. El conocimiento de su opinión parece, por ello, pertinente y relevante.

El objetivo de este trabajo ha sido conocer la opinión que los médicos con cierta experiencia en el uso de las TIC en la FMC tienen al respecto y, sobre todo, recabar las propuestas que estos profesionales realizan en el sentido de mejorar la eficiencia docente de las TIC en la formación médica.

\section{Sujetos y métodos}

\section{Sujetos}

Se ha realizado una encuesta a través de Internet que ha estado alojada en la página web de la Fundación Signo (http://www.fundacionsigno.com), dedicada al estudio de la gestión y la evaluación de costes sanitarios. Se ha remitido un correo electrónico a todos los miembros y seguidores de dicha fundación y a los médicos colegiados en el Colegio de Médicos de Toledo, con la petición de que contestaran dicha encuesta. Los datos sobre conocimiento de las TIC y sobre sus patrones de uso se han publicado previamente [13].

\section{Encuesta}

La encuesta consta de 24 preguntas (Google Drive) [14] cerradas de escalas de medición de actitudes y una respuesta abierta, utilizando la escala de valoración de Likert con cinco respuestas. El cuestionario consta de cinco partes: datos sociodemográficos y experiencia docente (cinco preguntas), experiencia en formación para médicos a través de Internet (cinco preguntas), experiencia en el uso de las TIC e Internet (tres preguntas), experiencia en el uso de las TIC en formación médica (nueve preguntas) y propuestas de mejora de la docencia médica a través de las TIC (dos preguntas). Se ha preguntado por las TIC teniendo en cuenta las aportaciones de Cabero y Román [15]. Las respuestas a las cuestiones relacionadas con el conocimiento y uso de las TIC se han publicado previamente [13], por lo que este trabajo se centra en las preguntas relacionadas con las propuestas de mejora de éstas.

Se realizó una evaluación por pares y un análisis de fiabilidad según el método de consistencia interna $\alpha$ de Cronbach para estudiar la validez del cuestionario, el cual se remitió a un grupo de expertos en metodología de la investigación, docencia y medicina al objeto de obtener sugerencias sobre él. Se valoró la idoneidad de las preguntas, la fluidez del cuestionario y su capacidad de discriminación, entre otros aspectos del diseño. Las correspondientes sugerencias y comentarios se introdujeron en el cuestionario definitivo. De nuevo, antes de remitir el cuestionario al total de los médicos encuestados, se realizó un estudio piloto enviándolo a un grupo aleatorio de 35 encuestados, seleccionados entre el grupo total de estudio, al objeto de realizar el análisis de consistencia interna según el $\alpha$ de Cronbach utilizando el programa estadístico Stata12. De estos registros se recibieron 26 respuestas, que fueron las incluidas en al análisis de fiabilidad. Con estos cuestionarios se obtuvo un $\alpha$ de Cronbach de 0,89, que se consideró adecuadó al ser mayor de 0,70, el límite considerado habitualmente como indicativo de adecuada fiabilidad. El $\alpha$ de Cronbach obtenido en cada uno de los módulos fue superior a 0,75 (entre $0,77$ y 0,90$)$. Tras comprobar la consistencia interna del cuestionario, se procedió a realizar su envío a todos los médicos colegiados.

\section{Análisis estadístico}

Los datos cualitativos se han expresado como promedio, y los cuantitativos, como media \pm desviación estándar o mediana más rango. Se han analizado las diferencias entre proporciones mediante la chi al cuadrado. Para el análisis estadístico se ha utilizado el programa Stata12. En las comparaciones de dos colas se ha considerado una $p<0,05$ como significativa. 
Tabla. Dificultades para el uso de las TIC en la formación médica continuada $(n=661)$.

\begin{tabular}{|c|c|c|c|c|c|c|}
\hline & Muchas & Bastantes & Regular & Pocas & Ninguna & $\begin{array}{l}\text { Muchas + } \\
\text { bastantes }\end{array}$ \\
\hline Dificultad de interacción entre alumno y profesor & $21(3,18 \%)$ & $135(20,42 \%)$ & $293(44,33 \%)$ & $179(27,08 \%)$ & $33(4,99 \%)$ & $23,60 \%$ \\
\hline Necesidad de constancia por parte del alumno para seguir el curso & $67(10,14 \%)$ & $323(48,87 \%)$ & $194(29,35 \%)$ & $68(10,29 \%)$ & $9(1,36 \%)$ & $59,00 \%$ \\
\hline Dificultad para evaluar adecuadamente el aprovechamiento del alumno & $27(4,08 \%)$ & $208(31,47 \%)$ & $286(44,78 \%)$ & $113(17,09 \%)$ & $27(4,08 \%)$ & $35,55 \%$ \\
\hline Otros (especificar) & $33(4,99 \%)$ & $74(9,68 \%)$ & $92(13,92 \%)$ & $52(7,87 \%)$ & $410(62,03 \%)$ & $16,19 \%$ \\
\hline Promedio & $38,4(5,81 \%)$ & $191,6(28,99 \%)$ & $220,6(33,37 \%)$ & $108,6(16,43 \%)$ & $101,8(15,40 \%)$ & $34,80 \%$ \\
\hline
\end{tabular}

\section{Resultados}

\section{Datos demográficos}

Se obtuvo la respuesta de 661 profesionales $(56,7 \%$ varones; $n=365$ ). La edad media era de 48,9 años. De los encuestados, 56 tenían una edad inferior a 35 años. El mayor porcentaje de respuestas $(n=202$; $30,6 \%)$ procedía de la provincia de Toledo, mientras que la procedencia del resto era variada ( $21 \%$ de Castilla y León, $10 \%$ de Madrid, $8 \%$ de Murcia, $4 \%$ de Cataluña...).

\section{Experiencia docente y laboral}

Un número elevado $(n=345 ; 52,19 \%)$ de los participantes son docentes, aunque también es alto el número de los que no lo son pero tienen experiencia o interés por el uso de las TIC como usuarios $(n=316 ; 47,81 \%)$. La experiencia en la docencia es amplia, aunque con claras variaciones: la mayoría tiene una experiencia docente mayor de $5(n=254$; $78 \%)$ o 10 años $(n=164 ; 50 \%)$.

La mayoría (61\%) de los médicos que respondieron realiza una labor asistencial, aunque no es despreciable el número de los que están en puestos directivos (16\%) y en labores no asistenciales (10\%). Aunque la mayoría trabaja en hospitales (50\%), hay también un número significativo que lo hace en atención primaria (34\%).

\section{Dificultades en el uso de las TIC para la FMC y su falta de difusión}

Al ser interrogados sobre las dificultades más importantes de los métodos de docencia médica a tra- vés de Internet, los encuestados consideran que la necesidad de constancia por parte del alumno (59\%) es la mayor de estas dificultades, seguido de la dificultad del idioma, dado que muchos cursos de calidad están en un idioma distinto al castellano (39,64\%). Las dificultades para evaluar adecuadamente el aprovechamiento del alumno y para realizar una interacción adecuada entre alumno y profesor son otras de las dificultades seleccionadas por los encuestados (Tabla).

La mayoría de los encuestados $(n=410$; $62 \%)$ considera que no hay una buena difusión de los métodos de docencia médica a través de Internet. Cuando son preguntados sobre las causas que ellos consideran que explican esta mala difusión, la mayoría $(42,7 \%)$ considera como responsable a la falta de hábito en el uso de estos métodos. Es de destacar que los participantes no consideran que la escasa difusión de las TIC en la docencia se deba a que sea aburrida la formación realizada mediante el uso exclusivo del ordenador $(7,3 \%)$, ni a que exista escasa interacción con el profesor durante la formación mediante estos cursos (5,4\%). Es decir, aunque sí ven en ésta una limitación de las TIC y sus sugerencias -como se indica más adelante- van encaminadas a mejorar esta comunicación, no consideran que este aspecto de dificultad en la comunicación sea un factor explicativo de la escasa difusión de los métodos.

\section{Estrategias para promover el uso de las TIC en la docencia}

A la hora de promover el uso de las TIC en la FMC, los participantes consideran que las mejores estrategias son el uso de publicidad en otros medios, 
Figura 1. Estrategias para mejorar la difusión de las TIC.

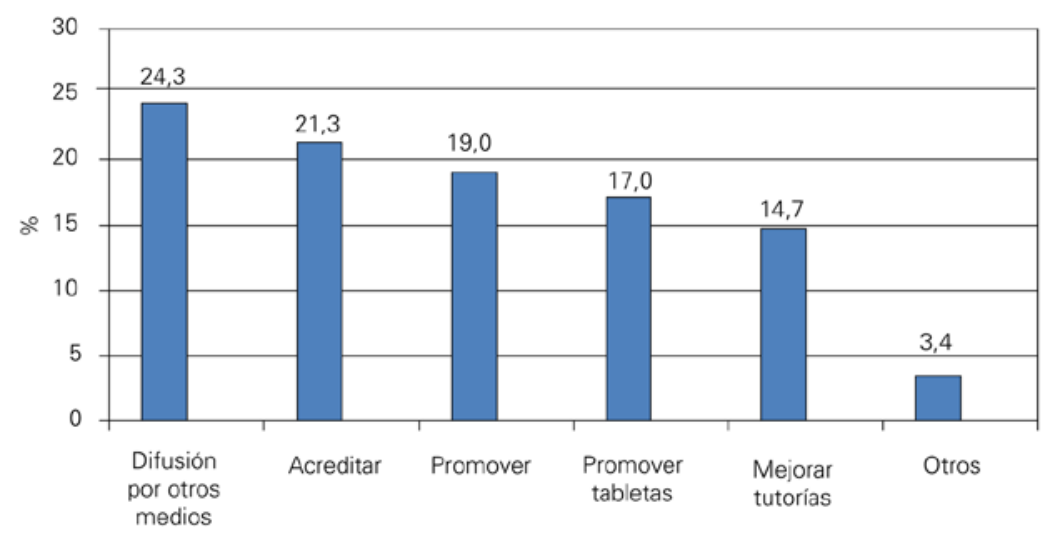

Figura 2. Limitaciones actuales de las TIC.

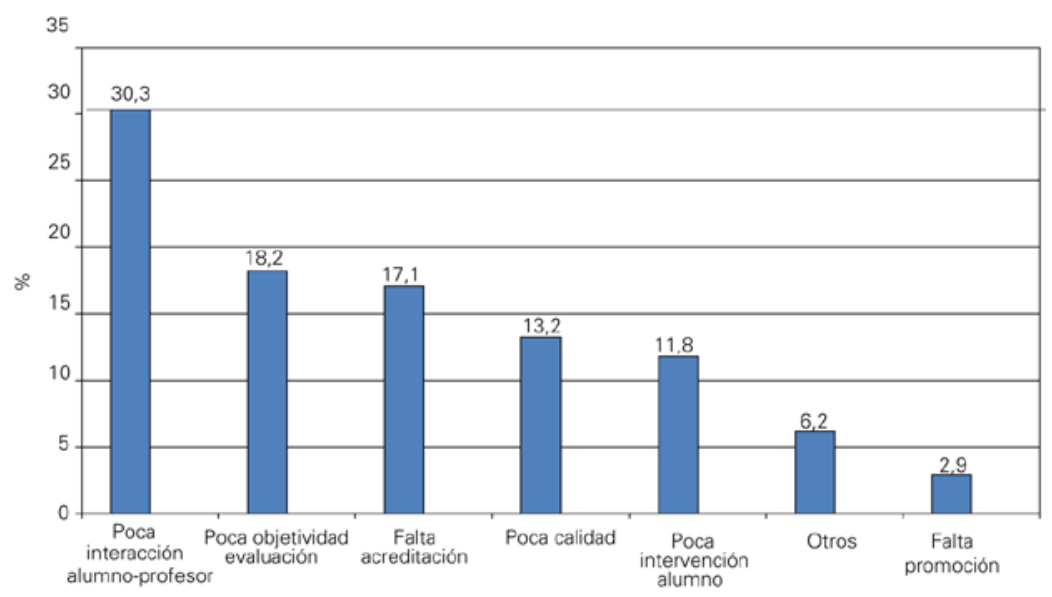

como los impresos (24,4\%), la acreditación oficial de las actividades formativas $(21,33 \%)$ y la promoción del uso de Internet entre los médicos (19,09\%). Quienes sugieren la promoción a través de otros medios distintos a Internet son significativamente más numerosos que los que sugieren la promoción del uso de Internet entre los médicos $(p=0,0195)$ o el uso de dispositivos del tipo tabletas digitales $(p=$ 0,0011) (Fig. 1).

\section{Limitaciones actuales de las TIC en la docencia}

Cuando se pregunta sobre las limitaciones de los mé- todos actuales de docencia a través de Internet, los encuestados indican que la más importante es la falta de interacción entre alumno y profesor $(n=$ $200 ; 30,3 \%$ ). Otros aspectos de los cursos, como la poca objetividad de la acreditación de los conocimientos adquiridos $(18,2 \%)$, la falta de acreditación de los cursos $(17,1 \%)$ y la poca intervención del alumno $(11,8 \%)$, tienen una importancia algo menor para los encuestados. Por el contrario, la falta de promoción por parte de la industria farmacéutica, que suele promocionar un buen número de estas actividades, tiene una relevancia menor $(2,9 \%)$ (Fig. 2).

\section{Recomendaciones para la mejora de las TIC en la docencia médica}

Las dos recomendaciones realizadas con mayor frecuencia hacen referencia a un mejor contacto e interacción entre el profesor y los alumnos, y de éstos entre sí: establecer un buen contacto alumno-profesor mejorando la tutoría $(21,8 \%)$ y promover mayor interacción del alumno durante el proceso de aprendizaje (20,4\%). Esto coincide con una de las limitaciones indicadas de la FMC a través de las TIC. Otras recomendaciones relevantes que indican los encuestados para facilitar el uso de las TIC en la FMC son la mejora de la calidad de los materiales docentes $(17,9 \%)$ y la consecución de acreditación de las actividades organizadas (13,6\%). La mejora de los sistemas de evaluación de aprendizaje en los cursos de FMC tiene también cierta relevancia para los encuestados (7,6\%). De igual forma, la obtención de respaldo por instituciones (sociedades científicas, universidades y autoridades sanitarias), aunque recomendada por una minoría (3,8$5,4 \%)$, lo es mucho más que el respaldo de los colegios de médicos $(0,9 \%)$ (Fig. 3).

En cuanto a las sugerencias de mejora, el número de encuestados que sugieren conseguir acreditación es significativamente menor que el de quienes sugieren mejorar el contacto con el profesor y la interacción entre los alumnos $(p=0,0001)$, o mejorar la calidad de los materiales que conseguir acreditación $(p=0,0344)$. Por el contrario, el número de quienes sugieren conseguir acreditación es significativamente mayor que el de los que indican que debería alcanzarse el respaldo de las sociedades científicas $(p=0,0003)$. Es de destacar que el número de quienes sugieren la obtención del respaldo de los colegios de médicos es significativamente menor que el de los que solicitarían el respaldo de sociedades científicas $(p<0,001)$ o de la universidad $(p=0,0006)$. 


\section{Discusión}

En este trabajo se recoge la opinión de los profesionales que utilizan las TIC en formación médica sobre las limitaciones de éstas, las estrategias para mejorar su difusión y las propuestas que realizan para mejorar su utilidad didáctica. La falta de interacción entre profesores y alumnos es la mayor limitación que los encuestados observan en las TIC $\mathrm{y}$, consecuentemente, una mejora de esta interacción y el desarrollo de unas actividades de mejor calidad son las propuestas de mejora más relevantes. Los datos obtenidos en este trabajo son especialmente importantes en un momento en el que se experimenta un notable incremento del desarrollo de herramientas TIC [16], pero en el que se carece de información sobre la opinión de los profesionales que deben utilizarlas, especialmente en el terreno de la FMC [6].

Existen algunos datos sobre el impacto de ciertas TIC en la docencia, especialmente en la Universidad de Edimburgo, donde desde los años noventa se ha analizado la experiencia, conocimiento y actitud ante las TIC de los nuevos estudiantes [17]. El informe SEUSSIS evaluó posteriormente el uso de las TIC en siete universidades europeas (Reino Unido, Finlandia, Noruega, Países Bajos, Italia, Francia y España), recogiendo datos de los años 2001-2002 [18], que se han añadido a otros especialmente en alumnos de medicina $[7,9,10,19]$ y profesionales de la salud [8], y han mostrado una situación muy similar a la descrita en nuestro trabajo. No obstante, no existe información sobre propuestas de mejora de estas herramientas para la docencia.

Una de las mayores tendencias actuales de la docencia de la medicina, al igual que en otros campos de la docencia, es la consolidación del uso de las TIC y la ampliación del aprendizaje a toda la labor de formación que debe llevar a cabo el individuo durante toda su vida [20], por lo que es fundamental conocer cómo consideran los encuestados que pueden desarrollarse mejores estrategias docentes basadas en las TIC y cómo deben diseñarse para que consigan su máxima eficacia.

Los encuestados valoran positivamente las ventajas de las TIC y consideran que sería útil mejorar la difusión de su uso, para lo cual sugieren diversas estrategias, entre las que destacan utilizar otros medios -como los escritos, por ejemplo-, acreditar adecuadamente los cursos y promover el uso de Internet entre los médicos y, especialmente, el de dispositivos que permitan una consulta más ubicua de este medio, como las tabletas digitales. Todas estas propuestas parecen razonables y dirigidas a dismi-
Figura 3. Sugerencias de mejora de las TIC.

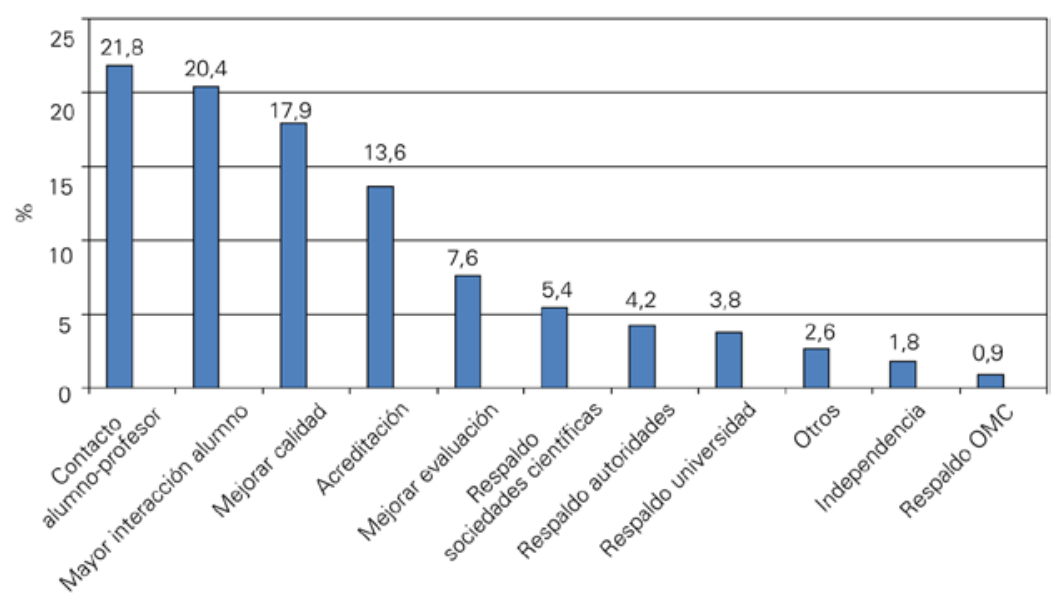

nuir la brecha digital existente en nuestro medio, como paso previo para obtener un mayor desarrollo y consolidación de las TIC en la docencia. Álvarez y López [21] comentan la importancia de desarrollar este tipo de estrategias para facilitar la implantación de las TIC, y especialmente el conocimiento que los docentes deben tener sobre ellas y sobre su uso en la docencia.

Con respecto a la mejora de la comunicación y de la interacción, la estrecha relación entre profesor y alumno es esencial para que ambos puedan alcanzar lo mejor del proceso de formación [22]. El alumno debe obtener información del profesor, con un feedback constructivo adecuado al tipo de enseñanza y al alumno, que le permita tener un conocimiento profundo y conveniente sobre sus acciones y sobre las consecuencias de éstas. Para el alumno, esta información es fundamental, tanto para continuar con la misma actitud si ésta es juzgada como positiva, como para reconducirla si, por el contrario, es contraproducente [23]; para el profesor, el feedback resulta esencial porque le permite demostrar su compromiso con el alumno en la consecución de los objetivos docentes. De hecho, se ha demostrado que la existencia de una interacción y feedback adecuados incrementa el tiempo dedicado a la formación, pero tiene un impacto positivo significativo en sus resultados [24].

Pero la relación entre alumno y profesor en medicina no es importante sólo por la obtención de feedback y por la evaluación, sino que resulta fundamental para el desarrollo de la tarea mentora y de 
tutoría, que desempeñan también un papel esencial en la docencia de la medicina $[25,26] \mathrm{y}$, de nuevo, benefician tanto al alumno como al profesor. A diferencia de lo que ocurre con un simple consejo, que se da de forma neutral, el mentor se implica y tiene un genuino interés en el éxito de la persona tutelada, que es la forma de medir el resultado de su acción a lo largo del tiempo. Queda claro que las personas que han participado en la encuesta sienten esta falta de comunicación como un déficit en la docencia a través de Internet que debe superarse, de forma que facilite las relaciones personales y permita el desarrollo de estas tareas docentes tan importantes como son la supervisión y la tutela.

Los encuestados requieren también una mayor interacción del alumno, entendida en un sentido más amplio y haciendo clara referencia a la relación con otros alumnos, lo que es sugerido por los encuestados como una de las mejoras propuestas para el desarrollo de las TIC en este terreno de la FMC. Esto se inscribe dentro de la tendencia hacia un aprendizaje más activo y de interrelación, que en medicina se traduce en aprendizaje en el equipo de trabajo e interactuando con todos los protagonistas de la asistencia, especialmente en grupos pequeños y con discusión de casos clínicos. Esta estrategia permite desarrollar una práctica más reflexiva y un pensamiento crítico, todo lo cual es fundamental para un razonamiento clínico adecuado [27].

Vemos, por tanto, que los encuestados valoran la relación y la conexión con sus compañeros como un elemento importante de la formación y en especial de la FMC, tal y como postula la teoría educativa de la conectividad, que considera esta relación como una elemento fundamental del aprendizaje innovador basado en las nuevas TIC [28]. Aunque este aspecto es más difícil de desarrollar, queda claro que los blogs y otras herramientas TIC pueden ayudar en este cometido [21,29]. Es fundamental un mayor conocimiento de estas herramientas, reconocido como limitado por los profesionales encuestados [13], si queremos conseguir una mejora de este aspecto tan importante de las TIC aplicadas a la FMC.

Otro aspecto relevante que señalan los profesionales encuestados es que debe elaborarse un material de calidad, es decir, debe utilizarse una metodología didáctica adecuada a las TIC y aprovechar la gran capacidad de interacción del material multimedia que ofrece esta tecnología. Meléndez [30] ha señalado que es fundamental que se produzcan cambios en los medios instruccionales con vistas a adaptarlos a las nuevas circunstancias en la formación mediante el uso de las TIC: sistema multimedia con peso importante de imagen y sonido, fo- mento de la participación e interactividad (comunidades virtuales), y transformación radial del espacio y del tiempo, de forma que el mensaje llegue directamente del emisor al receptor. Por todo ello, es importante realizar un diseño adecuado de la enseñanza o proceso de instrucción que trate de implementar estas propuestas de mejora [31].

Aunque es difícil asumir todos estos conceptos en el diseño de FMC con TIC, lo cierto es que puede seguirse una estrategia similar de presentación progresiva de la información a través de un caso para que, tras el análisis inicial, pueda proveerse la nueva información y ayudar a la integración de ésta. Un nuevo caso que obligue necesariamente a la incorporación de esa información ayudaría a integrar los nuevos conocimientos en la práctica habitual. De esta forma, la reflexión sobre los problemas debe ayudar a ir integrando lo aprendido en la práctica [32]. El desarrollo de foros y blogs para discutir el caso con los compañeros de aprendizaje, de forma tutelada por los instructores, puede aportar la interrelación y conectividad solicitadas por los encuestados y que es tan importante para un aprendizaje aprovechando la conectividad de las TIC. También se ha demostrado que estas herramientas pueden facilitar una evaluación más adecuada y razonable de los alumnos [29], lo que ha sido señalado también como una potencial limitación de las TIC por parte de los encuestados.

En la elaboración y presentación del material docente es importante seguir los principios de la teoría de la carga cognitiva, de manera que no se supere ninguna de las tres cargas cognitivas consideradas por Mayer [33]: intrínseca (realización de las múltiples funciones de la tarea que se está aprendiendo), extrínseca (resultado de tareas mal diseñadas que obligan a realizar más actividad de la estrictamente necesaria) y relevante (relacionado con el proceso que contribuye directamente al aprendizaje y a la formación de esquemas mentales). Según indica este autor, si el total de las cargas supera la capacidad de memoria activa del individuo, se entorpece el aprendizaje, por lo que las instrucciones formativas bien diseñadas deben minimizar la carga extrínseca y maximizar la carga relevante, lo que se consigue con un buen diseño del proceso. Como señala van Merriënboer [31], algunas estrategias del diseño del medio instruccional pueden ayudar a alcanzar este objetivo, por lo que conviene seguirlas.

En las revisiones sistemáticas realizadas sobre estrategias docentes basadas en Internet se ha observado que la existencia de interactividad, la repetición de los conceptos fundamentales, la realiza- 
ción de prácticas que permitan aplicar los conocimientos adquiridos y un feedback adecuado por parte del profesor tienen un impacto importante en los resultados docentes alcanzados [24].

Este estudio tiene algunas limitaciones que debemos señalar. En primer lugar, existe sesgo en la selección de la muestra dado que la encuesta se ha remitido sólo a médicos que utilizan el correo electrónico o Internet. De igual forma, la muestra no es aleatoria, a pesar de lo cual consideramos que el número de encuestas recibido parece representativo y puede ser razonable utilizar esta información en la mejora de las TIC porque, de momento, éstos son los profesionales que las utilizan.

En conclusión, los datos del estudio indican que debe mejorarse la interacción entre profesores y alumnos, así como entre los alumnos entre sí, al mismo tiempo que es fundamental desarrollar actividades de calidad técnica y pedagógica si queremos obtener una FMC basada en las TIC que presente la máxima eficiencia pedagógica.

\section{Bibliografía}

1. Prendes-Espinosa MP. Internet aplicado a la educación: estrategias didácticas y metodológicas. In Cabero-Almenara J, coord. Nuevas tecnologías aplicadas a la educación. Madrid McGraw-Hill; 2007. p. 205-22.

2. Gaikwad R, Warren J. The role of home-based information and communications technology interventions in chronic disease management: a systematic literature review. Health Informatics J 2009; 15: 122-46.

3. Landolina M, Perego GB, Lunati M, Curnis A, Guenzati G, Vicentini A, et al. Remote monitoring reduces healthcare use and improves quality of care in heart failure patients with implantable defibrillators: the evolution of management strategies of heart failure patients with implantable defibrillators (EVOLVO) study. Circulation 2012; 125: 2985-92.

4. Chang G, Cook D, Maguire T, Skakun E, Yakimets WW, Warnock GL. Problem-based learning: its role in undergraduate surgical education. Can J Surg 1995; 38: 13-21.

5. Nandi PL, Chan JN, Chan CP, Chan P, Chan LP. Undergraduate medical education: comparison of problem-based learning and conventional teaching. Hong Kong Med J 2000; 6: 301-6.

6. Gaglani SM, Toplol EJ. iMedEd: the role of mobile health technologies in medical education. Acad Med 2014; 89: 1207-9.

7. Dorup J. Experience and attitudes towards information technology among first-year medical students in Denmark: longitudinal questionnaire survey. J Med Internet Res 2004; 6: e10.

8. Jadad AR, Sigouin C, Cocking L, Booker L, Whelan T, Browman G. Internet use among physicians, nurse, and their patients. JAMA 2001; 286: 1451-2.

9. Gouveia-Oliveira A, Rodrigues T, De Melo FG. Computer education: attitudes and opinions of first-year medical students. Med Educ 1994; 28: 501-7.

10. Nurjahan MI, Lim TA, Foong A, Yeonf SW, Ware J. Computer literacy in medical students. Med Educ 2000; 34: 966.

11. Bravo-Toledo R. Contra la resistencia, innovación. Cibermedicina 2011; $1: 3$.

12. Rodríguez-Padial L, Cacheiro ML. Grado de conocimiento y aplicación de las nuevas tecnologías de la información y de la comunicación en la docencia de la electrocardiografía por los médicos de la provincia de Toledo. FEM 2014; 17: 21-30.

13. Rodríguez-Padial L, Cacheiro-González ML. Medina-Rivilla A. Conocimiento y uso de las tecnologías de la información y de la comunicación en la formación médica continuada. FEM 2015; 18: 283-91.

14. Alarco JJ, Álvarez-Andrade EV. Google Docs. Una alternativa de encuesta online. Educ Med 2012; 1: 9-10.

15. Cabero-Almenara J, Román-Graván P, coords. E-actividades. Un referente básico para la formación en Internet. Sevilla: Eduforma; 2005.

16. Sánchez J. El futuro de la mHealth: oportunidades de desarrollo para mantener prestaciones en épocas de crisis. El Médico 2014; 1150: 69-73.

17. Asgari-Jirhandeh N, Haywood J. Computer awareness among medical students: a survey. Med Educ 1997; 31: 225-9.

18. SEUSISS. Survey of European Universities Skills in ICT of Students and Staff; 2003.

19. Valcke M, De Wever, B. Information and communication technologies in higher education: evidence-based practices in medical education. Med Teach 2006; 28: 40-8.

20. Dent JA, Harden RM. New horizons in medical education. In Dent JA, Harden RM, eds. A practical guide for medical teaching. London: Churchill-Livingstone Elsevier; 2013. p. 3-7.

21. Álvarez G, López M. Análisis del uso de Facebook en el ámbito universitario desde la perspectiva del aprendizaje colaborativo a través de la computadora. EDUTEC Revista Electrónica de Tecnología Educativa 2013; 43: 1-15.

22. Krackov SK, Giving feedback. In Dent JA, Harden RM, eds. A practical guide for medical teaching. London: Churchill Livingstone Elsevier; 2013. p. 323-32.

23. Cohen D, Rhydderch M, Cooper I. Managing remediation. In Swanwick T, ed. Understanding medical education. Evidence, theory and practice. Oxford: Wiley Blackwell/ASME; 2014. p. 433-44.

24. Cook DA, Levinson AJ, Garside S, Dupras DM, Erwin PJ, Montori VM. Instructional design variations in internet-based learning for health professions education: a systematic review and meta-analysis. Acad Med 2010; 85: 909-22.

25. Ramani S, Gruppen L. Mentoring. In Dent JA, Harden RM, eds. A practical guide for medical teaching. London: Churchill Livingstone Elsevier; 2013. p. 142-8.

26. Launer J. Supervision, mentoring and coaching. In Swanwick T, ed. Understanding medical education. Evidence, theory and practice. Oxford: Wiley Blackwell/ASME; 2014. p. 111-22.

27. Pawlina W, Lachman N. Basic sciences and curriculum outcomes. In Dent JA, Harden RM, eds. A practical guide for medical teaching. London: Churchill Livingstone Elsevier; 2013. p. 240-5.

28. García IV. Theory of connectivity as an emergent solution to innovative learning strategies. Am J Educ Res 2014; 2: 107-16.

29. Molina P, Antolín L, Pérez-Samaniego V, Devís-Devís J, Villamón M, Valenciano J. Uso de blogs y evaluación continua del aprendizaje del alumnado universitario. EDUTEC Revista Electrónica de Tecnología Educativa 2013; 43: 1-13.

30. Meléndez R. Educación del siglo XXI mediada por las nuevas tecnologías de la información y comunicación, ¿qué cambios son necesarios? Revista de Tecnología de Información y Comunicación en Educación 2013; 7: 135-44.

31. Van Merriënboer JJG. Instructional design. In Dent JA, Harden RM, eds. A practical guide for medical teaching. London: Churchill Livingstone Elsevier; 2013. p. 199-206.

32. Muir F, Scott M, McConville K, Watson K, Behbehani K, Sukkar F. Taking the learning beyond the individual: how reflection informs change in practice. Int J Med Educ 2014; 5: 24-30.

33. Mayer RE. Applying the science of learning to medical education. Med Educ 2010; 44: 543-9. 